Dokuz Eylül University, adem.celik@deu.edu.tr, Izmir-Turkey

\title{
BİR FUL HİPERBOLİK BÖLGEDE EŞİTSİZLİKLER
}

\section{ÖZET}

$D \subset C$ sadece hiperbollerle sinırlı bir bölge (bir ful hiperbolik bölge) olsun. Bu bölgede, $f(0)=0, f(a)=a^{q}, f(-a)=-a^{q}$ olan $4 m$ dereceli kompleks değerli ünivalant polinom fonksiyonların $U T_{2,2}^{4} P(a)$ sınıfı için maksimum modül eşitsizlikleri elde edilmiştir. Bu eşitsizliklerde katsayılar: İlki $|a|^{\prime} y a$ bağlı olarak; bir diğeri polinomların sıfırlarına bağlı olarak ve üçünçüsü $|a|$ ya ve polinomların sıfırlarına bağlı olarak, üç farklı şekilde bulunmuştur. Sonra bu ful hiperbolik bölgenin bir özel durumu (Bir birim ful hiperbolik bölge) için eşitsizlikler elde edilmiştir.

Anahtar Kelimeler: Matematik Analiz, Öngörülen Üç Değeri Alan Ünivalant Fonksiyon, Hiperbolik Bölge, Maksimum Modül Değerler, Eşitsizlikler.

\section{INEQUALITIES IN A FULL HYPERBOLICAL REGION}

\section{ABSTRACT}

Let $D \subset C$ be a region bounded only by hyperbolas (a full hyperbolical region). In this region, we obtain maximum modulus inequalities for the class $U T_{2,2}^{4} P(a)$ of univalent polynomials functions with complex values of degree $4 m$, in which $f(0)=0, f(a)=a^{q}$, $f(-a)=-a^{q}$. The coefficients in these inequalities are obtained by three distinct ways: The firs is depending on $|a|$; the other one is depending on the zeros of polynomials, and the third is depending on $|a|$ and on the zeros of polynomials. Then we obtain inequalities for the special case where our region is a unit full hyperbolical region.

Keywords: Mathematical Analysis, Univalent Functions with Three Preassigned Values, Hyperbolical Region, Maximum Modulus Values, Inequalities. 


\section{GİRIŞ (INTRODUCTION)}

$D \subset C$ bölgesinde $f$ kompleks değerli ve kompleks değişkenli bir polinom fonksiyon olsun. $M_{f}=\max _{z \in \partial D}|f(z)|$ tanımlayalım. $M_{f_{1} \cdot f_{2} \ldots f_{n}}$ ile $M_{f_{1}} \cdot M_{f_{2}} \ldots M_{f_{n}}(n \geq 2)$ arasında eşitsizlikler $D$ bölgesi birim daire olduğunda [7, 14, 15 ve 17]'de, $D$ bölgesi $R \geq 1$ dairesi olduğunda [6]'da araştırılmıştır. Problem iki hiperbolik bölgede [4]'de ele alınmıştır ve

$B_{1}=\left\{z: \operatorname{Re}\left(z^{2}\right) \geq a^{2},-b \leq \operatorname{Re}(z) \leq b, b>a\right\}$, ve

$B_{2}=\left\{z: \operatorname{Re}\left(z^{2}\right) \leq a^{2}, \operatorname{Im}\left(-i z^{2}\right) \leq b^{2},-c \leq \operatorname{Im} z \leq c, c \geq b\right\}, \quad$ iki hiperbolik bölge olmak üzere aşağıdaki teoremler ispatlanmıştır:

Theorem A. Let $M_{f}=\max _{z \in \partial B_{i}}|f(z)|, \quad M_{g}=\max _{z \in \partial B_{i}}|g(z)|$ and $M_{f \cdot g}=\max _{z \in \partial B_{i}}|f(z) \cdot g(z)|$ be the maximum modulus values of the polynomial functions $f(z)=\prod_{i=1}^{m}\left(z-\alpha_{i}\right),\left(\alpha_{i} \neq 0\right), g(z)=\prod_{j=1}^{n}\left(z-\beta_{j}\right) \quad\left(\beta_{j} \neq 0\right)$, where $\min \left(\left|\alpha_{i}\right|,\left|\beta_{j}\right|\right)=r \quad(1 \leq i \leq m, 1 \leq j \leq n)$ on the hyperbolic regions $B_{i} \quad(i=1,2)$. (i) Let $a \neq r$ be on the hyperbolic region $B_{1}$. Then:

$$
M_{f \cdot g} \geq \delta \cdot M_{f} \cdot M_{g} \text { with } \delta=\left(\frac{|a-r|}{\sqrt{2 b^{2}-a^{2}}}\right)^{m+n} \cdot \frac{1}{2^{m}} \cdot \frac{1}{2^{n}} .
$$

(ii) Let $\min (a, b) \neq r$ be on the hyperbolic region $B_{2}$. Then:

$$
M_{f . g} \geq \theta \cdot M_{f} \cdot M_{g} \text { with } \theta=\left(\frac{|\min (a, b)-r|}{\sqrt{2 c^{2}+a^{2}}}\right)^{m+n} \cdot \frac{1}{2^{m}} \cdot \frac{1}{2^{n}} .
$$

Theorem B. Let $f(z)=z^{k} \prod_{i=1}^{m}\left(z-\alpha_{i}\right),\left(\alpha_{i} \neq 0, k \leq m\right), \quad g(z)=z^{p} \prod_{j=1}^{n}\left(z-\beta_{j}\right)$ $\left(\beta_{j} \neq 0, p \leq n\right)$ be polynomials on the hyperbolic regions $B_{i}(i=1,2)$, satisfiying $\min \left(\left|\alpha_{i}\right|,\left|\beta_{j}\right|\right)=r \quad(1 \leq i \leq m-k, \quad 1 \leq j \leq n-p)$.

(i) Let $a \neq r$ be on the hyperbolic region $B_{1}$. Then:

$$
M_{f . g} \geq \varepsilon_{2} \cdot M_{f} \cdot M_{g} \text { where } \varepsilon_{2}=\left(\frac{a}{\sqrt{2 a^{2}-b^{2}}}\right)^{k+p} \cdot\left(\frac{|a-r|}{\sqrt{2 b^{2}-a^{2}}}\right)^{m+n-k-p} \cdot \frac{1}{2^{m-k}} \cdot \frac{1}{2^{n-p}}
$$

(ii) Let $\min (a, b) \neq r$ be on the hyperbolic region $B_{2}$. Then:

$$
M_{f . g} \geq \varepsilon_{3} \cdot M_{f} \cdot M_{g} \text { where } \varepsilon_{3}=\left(\frac{\min (a, b)}{\sqrt{2 c^{2}+a^{2}}}\right)^{k+p} \cdot\left(\frac{|\min (a, b)-r|}{\sqrt{2 c^{2}+a^{2}}}\right)^{m+n} \cdot \frac{1}{2^{4 m}} \cdot \frac{1}{2^{4 n}}
$$

$\mathrm{Bu}$ teoremler $n$ tane polinom fonksiyon için de genelleştirilmiştir. Ayrıca bazı özel durumlar için de formüller elde edilmiştir. [5]'de $\rho \in C, B=\{z \in C:|z|<r, 0<r<\infty\}$ ve $n=1,2, \ldots, N \quad(N$ sonlu) olmak üzere, $0,-a, a$ reel noktalarını sabit bırakan $F_{n}: D \rightarrow C$, 
$\overline{F_{n}(z)=z+\rho\left(a^{2 n} z^{2 n}-z^{4 n}\right), 0<a<r \quad \text { ünivalant fonksiyonların sinıfını }}$ $\left\{F_{n}(a: r)\right\}$ ile ve $S_{n}: D \rightarrow C, S_{n}(z)=z+\rho \sum_{k=1}^{n}\left(a^{2 k} z^{2 k}-z^{4 k}\right), 0<a<r$ ünivalant fonksiyonların sınıfı da $\left\{S_{n}(a: r)\right\}$ ile belirtilmiştir. $D$ açık diskinin otomorfizmaları araştırılmıştır.[3]'de $0, a,-a \in \bar{D} \quad$ ve $q \in N$ olmak üzere $f(0)=0, f(a)=a^{q}, f(-a)=-a^{q}$ olan ünivalant polinom fonksiyonların kümesi $\operatorname{UTP}(a)$ ile tanımlanmıştır.UTP $(a)$ içinde derecesi $4 m$ ve $q \in\{1,3,5, \ldots, 4 m-1\} \quad$ olmak üzere, $\quad f(z)=z^{q}+\sum_{k=1}^{m} \rho_{k}\left(z^{4 k}-a^{2 k} z^{2 k}\right)$ $\left(\rho_{k} \in C, \rho_{m}=1\right)$ formundaki tüm polinom fonksiyonların sınıfı da $U T_{2,2}^{4} P(a)$ ile belirtilmiştir. Bu sınıf için hem birim hem de herhangi bir $R<\infty$ yarıçaplı bir diskte $M_{f_{1} \cdot f_{2} \ldots f_{n}}$ ile $M_{f_{1}} \cdot M_{f_{2}} \ldots M_{f_{n}}(n \geq 2)$ arasında eşitsizlikler araştırılmıştır. Benzer sınıflar $U T_{3,1}^{4} P(a)$, $U T_{1,3}^{4} P(a), U T_{4,0}^{4} P(a)$ ile tanımlanmıştır.

TANIM 1: Bir $A \subset C$ bölgesinin sinırı tamamen hiperbol eğrilerinden oluşuyorsa $A$ bölgesi bir "ful hiperbolik bölgedir" denir.

TANIM 2: Bir ful hiperbolik bölgenin tüm hiperbol eğrilerinin tepe noktaları birim çembere teğet oluyorsa, bu hiperbolik bölgeye "bir birim ful hiperbolik bölgedir" diyelim.

Şimdi $a_{0}, b_{0}, c_{0}, d_{0}$ pozitif reel sayllar olsun. $\operatorname{Re}\left(z^{2}\right)=a_{0}^{2}$, $\operatorname{Im}\left(-i z^{2}\right)=b_{0}^{2}, \quad \operatorname{Im}\left(z^{2}\right)=2 c_{0}^{2}, \quad \operatorname{Im}\left(-z^{2}\right)=2 d_{0}^{2} \quad\left(i^{2}=-1\right)$ hiperbol eğrilerini göz önüne alalım. Bu hiperbol eğrileri ile sınırlı bölgeyi $D$ ile gösterelim. $D$ bölgesi bir ful hiperbolik bölgedir.

ÇapD $=2 d$ alalım. $D$ bölgesinin hiperbol eğrilerinin birbirini kestiği noktaları $E_{j}(1 \leq j \leq 8)$ ile gösterelim. $E_{j}, 4$ kesim noktasından geçen ve $D$ bölgesini kapsayan dairenin çap uzunluğu da $2 d$ dir. Açık şekilde Maks $(|z-w|: z, w \in \bar{D}) \leq 2 d$ dir. Burada, $\partial D$ simgesi $D^{\prime}$ nin sınırını belirtmek üzere $\bar{D}=\partial D \cup D$ dir.

\section{2. ÇALIŞMANIN ÖNEMİ (REASERCH SIGNIFICANCE)}

$f, g$ kompleks değerli ve kompleks değişkenli iki polinom fonksiyonunu göz önüne alalım. $z$ kompleks değişken olsun. Birim diskte $M_{f}=\max _{|z|=1}|f(z)|, M_{g}=\max _{|z|=1}|g(z)|$ ve $M_{f \cdot g}=\max _{|z|=1}|f(z) \cdot g(z)|$, herhangi bir $R<\infty$ yarıçaplı bir disk için ise $M_{f}^{\prime}=\max _{|z|=R}|f(z)|, \quad M_{g}^{\prime}=\max _{|z|=R}|g(z)| \quad$ ve $M_{f . g}^{\prime}=\max _{|z|=R}|f(z) \cdot g(z)|$ tanımlayalım. $M_{f . g}$ ile $M_{f} \cdot M_{g}$ arasında [3, 7, 12, 14, 15 ve 17]'da ve herhangif, $g$ polinomları için [6]'da eşitsizlikler, $f, g \in U T_{2,2}^{4} P(a)$ polinomları için $M_{f . g}^{\prime}$ ile $M_{f}^{\prime} \cdot M_{g}^{\prime}$ arasında yeni eşitsizlikler [3]'de bulunmuştur. İk defa ilgili problem hiperbolik 
bölgelerde [4]'de ele alınmış ve benzer yeni eşitsizlikler elde edilmiştir.

Bu çalışmada ilgili problem bir ful hiperbolik bölgede (tamamen hiperbollerle sınırlı bir bölgede) ele alınarak $U T_{2,2}^{4} P(a)$ sınıfı için eşitsizlikler bulunmuştur. Ayrıca bir birim ful hiperbolik bölge tanımlanarak bu bölge için de eşitsizlikler elde edilmiştir.

\section{ANALITIKK ÇALIŞMA (ANALYTICAL STUDY)}

Teorik metotlarla elde edilen bağıntı veya denklemlere "analitik bağıntılar" denir. Pür çalışmalarda ispat teknikleri aracılığıyla teorik metotlar kullanılarak bağıntılar (formüller) veya denklemler elde edilir. Bir analitik bağıntıyı bulmak için uygulanan bu teorik metotlar genellikle Teoerem-Hipetez-Hüküm ve ispat çerçevesinde şekillenir [3].

Çalışmamız pür matematik tabanlı bir analitik çalışmadır ve ispatlarda [3 ve 4] deki ispat yöntemlerinden kısmen yararlanılmıştır.

\section{4. $D^{\prime} \mathrm{DE} U T_{2,2}^{4} P(a)$ İÇİN MAKSİMUM MODÜL EŞİTSİZLİKLERİ} (MAXIMUM MODULUS INEQUALITIES FOR $U T_{2,2}^{4} P(a)$ ON $D$ )

Bu kesimde ful hiperbolik $D$ bölgesinde $U T_{2,2}^{4} P(a)$ sınıfı için eşitsizlikler vereceğiz. Önce aşağıdaki Lemma'yı ispatlayalım.

Lemma 1. Ful hiperbolik bölge $D$ olsun. O zaman her $z \in \partial D$ için $|z| \geq \min \left(a_{0}, b_{0}, \sqrt{2} \cdot c_{0}, \sqrt{2} . d_{0}\right)$ dir.

İspat. $K_{1}=\left\{z \in C: \operatorname{Im}\left(z^{2}\right)=2 c_{0}^{2}\right\}$ kümesini, $K_{2}=\left\{z \in C: \operatorname{Im}\left(-z^{2}\right)=2 d_{0}^{2}\right\}$, $K_{3}=\left\{z \in C: \operatorname{Im}\left(-i z^{2}\right)=b_{0}^{2}\right\} \quad$ kümesini ve $K_{4}=\left\{z \in C: \operatorname{Re}\left(z^{2}\right)=a_{0}^{2}\right\} \quad$ kümesini ele alalım. Önce her $z \in K_{1}$ için $|z| \geq \sqrt{2} \cdot c_{0}$ olduğunu gösterelim. Bunun için $z=|z| \cdot e^{i \theta} \in K_{1}$ alalım. Bu durumda ya $0<\theta<\frac{\pi}{2} \quad$ ya da $\pi<\theta<\frac{3 \pi}{2}$ dir. Eğer $0<\theta<\frac{\pi}{2}$ ise $0<2 \theta<\pi$; eğer $0<\frac{\pi}{2}<\theta<\pi$ ise $\pi<2 \theta<2 \pi$ olur. Öte yandan her $z \in K_{1}$ için $|z|^{2} \cdot \sin 2 \theta=2 c_{0}^{2}$ dir. Buradan, $|z|^{2} \geq 2 c_{0}^{2}$ ya da $|z| \geq \sqrt{2} . c_{0}$ bulunur. Benzer kümeler için aynı ispatı yaparak, her $z \in K_{2}$ için $|z| \geq \sqrt{2} \cdot d_{0}$, her $z \in K_{3}$ için $|z| \geq b_{0}$ ve her $z \in K_{4} \quad$ için $|z| \geq a_{0}$ bulunur. Ful hiperbolik bölge $D$ göz önüne alınırsa, her $z \in \partial D$ için $|z| \geq \min \left(a_{0}, b_{0}, \sqrt{2} \cdot c_{0}, \sqrt{2} \cdot d_{0}\right)$ olduğu görülür.

\section{1. $|a|^{\prime}$ ya Bağlı Eşitsizlikler (Inequalities Depending on $|a|$ )}

Bu alt kesimde $D$ bölgesinde $U T_{2,2}^{4} P(a)$ sınıfı için $|a|^{\prime}$ ya bağlı eşitsizlikler elde edeceğiz.

Teorem 1. $D$ Bölgesinde $4 m$ ve $4 n$ surasıyla $f, g \in U T_{2,2}^{4} P(a)$ polinom fonksiyonlarının dereceleri ve $f(\mp a)=\mp a^{p}, \quad g(\mp a)=\mp a^{q}$ ve $\forall \rho_{1} \neq 0$ olsun. Bu halde,

(i) $\min (p, q) \geq 3$ ise, 


$$
M_{f \cdot g} \geq \theta_{1} \cdot M_{f} \cdot M_{g}
$$

burada $\theta_{1}=\left[\min \left(a_{0}, b_{0}, \sqrt{2} \cdot c_{0}, \sqrt{2} \cdot d_{0}\right)\right]^{4} \cdot \frac{|a|^{p+q-4}}{d^{4 m+4 n}} \cdot \frac{1}{2^{4 m-2}} \cdot \frac{1}{2^{4 n-2}}$.

(ii) $p=q=1$ (ya da $0,-a, a$ noktaları polinomların sabit noktaları) ise,

$$
M_{f . g} \geq \theta_{2} \cdot M_{f} \cdot M_{g}
$$

burada $\theta_{2}=\left[\min \left(a_{0}, b_{0}, \sqrt{2} \cdot c_{0}, \sqrt{2} \cdot d_{0}\right)\right]^{2} \cdot \frac{1}{d^{4 m+4 n}} \cdot \frac{1}{2^{4 m-1}} \cdot \frac{1}{2^{4 n-1}}$.

(iii) $\min (p, q)=1$ ise,

$$
M_{f . g} \geq \theta_{3} \cdot M_{f} \cdot M_{g}
$$

burada $\theta_{3}=\left[\min \left(a_{0}, b_{0}, \sqrt{2} \cdot c_{0}, \sqrt{2} \cdot d_{0}\right)\right]^{2} \cdot \frac{|a|^{p+q-2}}{d^{4 m+4 n}} \cdot \frac{1}{2^{4 m-1}} \cdot \frac{1}{2^{4 n-1}}$.

İspat. (i) $h_{1}, h_{2}$ fonksiyonları sirasiyla

$$
\begin{aligned}
& h_{1}(z)=z^{p-2}+\rho_{1}\left(z^{2}-a^{2}\right)+\rho_{2}\left(z^{6}-a^{4} \cdot z^{2}\right)+\ldots+\left(z^{4 m-2}-a^{2 m} \cdot z^{2 m-2}\right), \\
& h_{2}(z)=z^{q-2}+\rho_{1}^{\prime}\left(z^{2}-a^{2}\right)+\rho_{2}^{\prime}\left(z^{6}-a^{4} \cdot z^{2}\right)+\ldots+\left(z^{4 n-2}-a^{2 n} \cdot z^{2 n-2}\right)
\end{aligned}
$$

formunda iki polinom olsun. $f, g \in U T_{2,2}^{4} P(a)$ fonksiyonları sirasıyla,

$$
\begin{aligned}
& f(z)=z^{2} \cdot h_{1}(z) \text { ve } g(z)=z^{2} \cdot h_{2}(z) \text { formunda yazılabilir. O zaman } \\
& |f(z)|=|z|^{2} \cdot\left|h_{1}(z)\right| \text { ve }|g(z)|=|z|^{2} \cdot\left|h_{2}(z)\right| \text { olduğudan, her } z \in \bar{D} \text { için } \\
& |f(z)| \leq d^{2} \cdot(2 d)^{4 m-2} \text { ve }|g(z)| \leq d^{2} \cdot(2 d)^{4 n-2} \text { ve buradan da, } \\
& \quad M_{f}=\max _{z \in D D}|f(z)| \leq d^{2} \cdot(2 d)^{4 m-2} \text { ve } M_{g}=\max _{z \in D D}|g(z)| \leq d^{2} \cdot(2 d)^{4 n-2} \text { olur. } \\
& \quad \text { zaman, } \\
& \quad M_{f} \cdot M_{g}=\max _{z \in D D}|f(z)| \cdot \max _{z \in D D}|g(z)| \leq d^{4} \cdot(2 d)^{4 m-2+4 n-2}=2^{4 m-2} \cdot 2^{4 n-2} \cdot d^{4 m+4 n}
\end{aligned}
$$

bulunur. Öte yandan, Lemma 1 gereğince her $z \in \partial D$ için sırasıyla,

$|f(z)| \geq\left(\min \left(a_{0}, b_{0}, \sqrt{2} \cdot c_{0}, \sqrt{2} \cdot d_{0}\right)\right)^{2} \cdot\left|h_{1}(z)\right|$,

$\left.|g(z)| \geq\left(\min \left(a_{0}, b_{0}, \sqrt{2} \cdot c_{0}\right), \sqrt{2} \cdot d_{0}\right)\right)^{2} \cdot\left|h_{2}(z)\right|$, olduğundan

$|f(z) \cdot g(z)| \geq\left(\min \left(a_{0}, b_{0}, \sqrt{2} \cdot c_{0}, \sqrt{2} \cdot d_{0}\right)\right)^{4} \cdot\left|h_{1}(z) \cdot h_{2}(z)\right|$ ve buradan

$\max _{z \in \partial D}|f(z) \cdot g(z)| \geq \max _{z \in \partial D}\left(\left(\min \left(a_{0}, b_{0}, \sqrt{2} \cdot c_{0}, \sqrt{2} \cdot d_{0}\right)\right)^{4} \cdot\left|h_{1}(z) \cdot h_{2}(z)\right|\right) \quad$ yazarız. Simdi, $\left|H_{1}(z)\right|=\min \left(a_{0}, b_{0}, \sqrt{2} \cdot c_{0}, \sqrt{2} \cdot d_{0}\right)^{4} \cdot\left|h_{1}(z) \cdot h_{2}(z)\right|$ alalım.

$\left|H_{1}(a)\right|=\left(\min \left(a_{0}, b_{0}, \sqrt{2} \cdot c_{0}, \sqrt{2} \cdot d_{0}\right)\right)^{4} \cdot|a|^{p-2} \cdot|a|^{q-2}$ olduğu için, maximum modul prensibi gereğince, $\max _{z \in C D}\left|H_{1}(z)\right|>\left(\min \left(a_{0}, b_{0}, \sqrt{2} \cdot c_{0}, \sqrt{2} . d_{0}\right)\right)^{4} \cdot|a|^{p-2} \cdot|a|^{q-2}$ elde edilir. Buradan da,

$$
M_{f . g}=\max _{z \in \partial D}|f(z) \cdot g(z)| \geq\left(\min \left(a_{0}, b_{0}, \sqrt{2} \cdot c_{0}, \sqrt{2} \cdot d_{0}\right)\right)^{4} \cdot|a|^{p-2} \cdot|a|^{q-2}
$$

bulunur. (8) ve (9) den (7) elde edilir. (ii) $f, g \in U T_{2,2}^{4} P(a)$ fonksiyonları sırasıyla, $h_{3}, h_{4}$ polinom fonksiyonları $4 m-1,4 n-1$ dereceli olmak üzere $f(z)=z \cdot h_{3}(z), g(z)=z \cdot h_{4}(z)$ formunda yazılabilir 
ve $h_{3}(a) \cdot h_{4}(a)=1$ dir. (iii) $f, g \in U T_{2,2}^{4} P(a)$ fonksiyonları sirasıyla, $h_{5}, h_{6}$ polinom fonksiyonları $4 m-1,4 n-1$ dereceli olmak üzere $f(z)=z \cdot h_{5}(z)$, $g(z)=z \cdot h_{6}(z)$ formunda yazılabilir ve $h_{5}(a) \cdot h_{6}(a)=a^{q-1} \cdot a^{p-1}$ dir. Bu iki hal için (i) deki ispat yolu izlenir.

Teorem 2. (Genelleştirilmiş Teorem 1) $n \geq 2$ olsun. $D$ Bölgesinde $4 m_{1}, 4 m_{2}, \ldots, 4 m_{n}$ sirasiyla $f_{1}, f_{2}, \ldots, f_{n} \in U T_{2,2}^{4} P(a)$ polinom fonksiyonlarının dereceleri olmak üzere, $f_{i}(\mp a)=\mp a^{q_{i}}$ ve $\forall \rho_{1} \neq 0(i=1,2, . ., n)$ olsun. O zaman, (i) $\min \left(q_{1}, q_{2}, \ldots, q_{n}\right) \geq 3$ ise,

$$
\theta_{1}^{\prime}=\left[\min \left(a_{0}, b_{0}, \sqrt{2} \cdot c_{0}, \sqrt{2} \cdot d_{0}\right)\right]^{2 n} \cdot \frac{|a|^{q_{1}+q_{2}+\ldots+q_{n}-2 n}}{d^{4 m_{1}+4 m_{2}+\ldots .+4 m_{n}}} \cdot \frac{1}{2^{4 m_{1}-2}} \cdot \frac{1}{2^{4 m_{m}-2}} \ldots \frac{1}{2^{4 m_{n}-2}}
$$

olmak üzere

$$
M_{f_{1} \cdot f_{2} \cdots f_{n}} \geq \theta_{1}^{\prime} \cdot M_{f_{1}} \cdot M_{f_{2}} \ldots M_{f_{n}} \text { dir. }
$$

(ii) $q_{1}=q_{2}=\ldots=q_{n}=1$ (ya da $0,-a, a$ noktaları polinomların sabit noktaları) ise,

olmak üzere

$$
\theta_{2}^{\prime}=\left[\min \left(a_{0}, b_{0}, \sqrt{2} \cdot c_{0}, \sqrt{2} \cdot d_{0}\right)\right]^{n} \cdot \frac{1}{d^{4 m_{1}+4 m_{2}+\ldots+4 m_{n}}} \cdot \frac{1}{2^{4 m_{1}-1}} \cdot \frac{1}{2^{4 m_{m}-1}} \ldots \frac{1}{2^{4 m_{n}-1}}
$$

$$
M_{f_{1} \cdot f_{2}} \cdots f_{n} \geq \theta_{2}^{\prime} \cdot M_{f_{1}} \cdot M_{f_{2}} \ldots M_{f_{n}} \operatorname{dir} .
$$

(iii) $\min \left(q_{1}, q_{2}, \ldots, q_{n}\right)=1$ ise,

olmak üzere

$$
\theta_{3}^{\prime}=\left[\min \left(a_{0}, b_{0}, \sqrt{2} \cdot c_{0}, \sqrt{2} \cdot d_{0}\right)\right]^{n} \cdot \frac{|a|^{q_{1}+q_{2}+\ldots+q_{n}-n}}{d^{4 m_{1}+4 m_{2}+\ldots+.4 m_{n}}} \cdot \frac{1}{2^{4 m_{1}-1}} \cdot \frac{1}{2^{4 m_{m}-1}} \ldots \frac{1}{2^{4 m_{n}-1}}
$$

$$
M_{f_{1} \cdot f_{2}} \cdots f_{n} \geq \theta_{3}^{\prime} \cdot M_{f_{1}} \cdot M_{f_{2}} \ldots M_{f_{n}} \text { dir. }
$$

Sonuç Teorem 1. $n \geq 2$ olsun. $D$ Bölgesinde $4 m_{1}, 4 m_{2}, \ldots, 4 m_{n}$ sirasıyla $f_{1}, f_{2}, \ldots, f_{n} \in U T_{2,2}^{4} P(a)$ polinom fonksiyonlarının dereceleri olmak üzere, $f_{i}(\mp a)=\mp a^{q_{i}}$ ve $\forall \rho_{1} \neq 0(i=1,2, . ., n) \circ l$ sun. $j \leq n$ tane $f_{1}, f_{2}, \ldots, f_{j}$ için $\min \left(q_{1}, q_{2}, \ldots, q_{j}\right) \geq 3$, geriye kalan $n-j$ tane $f_{j+1}, f_{j+2}, \ldots$, $f_{n}$ için $\min \left(q_{j+1}, q_{j+2}, \ldots, q_{n}\right)=1$ ise,

$$
\begin{aligned}
\theta_{4}=\left[\min \left(a_{0}, b_{0}, \sqrt{2} \cdot c_{0}, \sqrt{2} \cdot d_{0}\right)\right]^{2 j+(n-j)} \cdot \frac{|a|^{q_{1}+q_{2}+\ldots+q_{n}-(2 j+(n-j))}}{d^{4 m_{1}+4 m_{2}+\ldots+4 m_{n}}} \\
\cdot \frac{1}{2^{4 m_{1}-2}} \cdot \frac{1}{2^{4 m_{m}-2}} \ldots \frac{1}{2^{4 m_{j}-2}} \cdot \frac{1}{2^{4 m_{j+1}-1}} \cdot \frac{1}{2^{m_{j+2}-1}} \ldots \frac{1}{2^{4 m_{n}-1}}
\end{aligned}
$$

olmak üzere

$$
M_{f_{1} \cdot f_{2}} \cdots f_{n} \geq \theta_{4} \cdot M_{f_{1}} \cdot M_{f_{2}} \cdots M_{f_{n}} \operatorname{dir} .
$$




\subsection{Polinomların Sıfırlarına Bağlı Eşitsizlikler (Inequalities Depending on the Zeros of Polynomials)}

Bu alt kesimde $D$ bölgesinde $U T_{2,2}^{4} P(a)$ sınıfı için polinomların köklerine bağlı eşitsizlikler elde edeceğiz.

Teorem 3. $D$ Bölgesinde $4 m$ ve $4 n$ sirasiyla $f, g \in U T_{2,2}^{4} P(a)$ polinom fonksiyonlarının dereceleri ve $f(\mp a)=\mp a^{p}, \quad g(\mp a)=\mp a^{q}$, $\forall \rho_{1} \neq 0 ; \quad \alpha_{i} \neq 0 \quad(1 \leq i \leq 4 m-2) \quad f^{\prime}$ nin sifirları, $\quad \beta_{i} \neq 0 \quad(1 \leq j \leq 4 n-2)$ $g$ 'nin siflrları olsun. Bu halde,

$$
\begin{aligned}
\theta_{5}= & {\left[\min \left(a_{0}, b_{0}, \sqrt{2} \cdot c_{0}, \sqrt{2} \cdot d_{0}\right)\right]^{4} \cdot \frac{\prod_{i=1}^{4 m-2}\left|\alpha_{i}\right| \cdot \prod_{j=1}^{4 n-2}\left|\beta_{j}\right|}{d^{4 m+4 n}} \cdot \frac{1}{2^{4 m-2}} \cdot \frac{1}{2^{4 n-2}} \text { olmak üzere, } } \\
& M_{f . g} \geq \theta_{5} \cdot M_{f} \cdot M_{g} \operatorname{dir} .
\end{aligned}
$$

İspat.Hipotezden, $f$ ve $g$ polinom fonksiyonları sırasıyla $f(z)=z^{2} \cdot \prod_{i=1}^{4 m-2}\left(z-\alpha_{i}\right)$ ve $g(z)=z^{2} \cdot \prod_{i=1}^{4 n-2}\left(z-\beta_{i}\right)$ biçiminde yazılabilir. Şimdi $h_{11}, h_{22}$ fonksiyonları sirasıyla $h_{11}(z)=\prod_{i=1}^{4 m-2}\left(z-\alpha_{i}\right)$ ve $h_{22}(z)=$ $\prod_{j=1}^{4 n-2}\left(z-\beta_{j}\right)$ ise, $\left|h_{11}(0)\right|=\prod_{i=1}^{4 m-2}\left|\alpha_{i}\right|$ ve $\left|h_{22}(0)\right|=\prod_{j=1}^{4 n-2}\left|\beta_{j}\right|$ olur. o zaman $\left|h_{11}(0) \cdot h_{22}(0)\right|=\prod_{i=1}^{4 m-2}\left|\alpha_{i}\right| \cdot \prod_{j=1}^{4 n-2}\left|\beta_{j}\right|$ elde edilir. Ĕğer, $H(z)=\prod_{i=1}^{4 m-2}\left(z-\alpha_{i}\right) \cdot \prod_{j=1}^{4 n-2}\left(z-\beta_{j}\right)$ tanımlarsak $\max _{z \in \partial D}|H(z)|>\prod_{i=1}^{4 m-2}\left|\alpha_{i}\right| \cdot \prod_{j=1}^{4 n-2}\left|\beta_{j}\right|$ olur. Öte yandan $|f(z) \cdot g(z)|=|z|^{4} \cdot|H(z)|$ olduğu için Lemma 1 gereğince her $z \in \partial D$ için $|f(z) \cdot g(z)| \geq\left[\min \left(a_{0}, b_{0}, \sqrt{2} \cdot c_{0}, \sqrt{2} \cdot d_{0}\right)\right]^{4} \cdot|H(z)|$ ve buradan da

$$
M_{f . g}=\max _{z \in \partial D}|f(z) \cdot g(z)| \geq\left[\min \left(a_{0}, b_{0}, \sqrt{2} \cdot c_{0}, \sqrt{2} \cdot d_{0}\right)\right]^{4} \cdot \prod_{i=1}^{4 m-2}\left|\alpha_{i}\right| \cdot \prod_{j=1}^{4 n-2}\left|\beta_{j}\right|
$$

bulunur. Ayrıca her $z \in \bar{D}$ için $|z| \leq d,\left|z-\alpha_{i}\right| \leq 2 d$ ve $\left|z-\beta_{j}\right| \leq 2 d$ olduğundan

$$
M_{f} \cdot M_{g}=\max _{z \in \partial D}|f(z)| \cdot \max _{z \in \partial D}|g(z)| \leq 2^{4 m-2} \cdot 2^{4 n-2} \cdot d^{4 m+4 n}
$$

bulunur. (15) ve (16) dan da (14) Formülü elde edilir.

Teorem 4. (Genelleştirilmiş Teorem 3) $n \geq 2$ olsun. $D$ Bölgesinde $4 m_{1}, 4 m_{2}, \ldots, 4 m_{n} \quad$ sirasiyla $\quad f_{1}, f_{2}, \ldots, f_{n} \in U T_{2,2}^{4} P(a) \quad$ polinom fonksiyonlarının dereceleri ve $f_{i}(\mp a)=\mp a^{q_{i}}, \quad \forall \rho_{1} \neq 0(i=1,2, . ., n)$; $\alpha_{i 1} \neq 0\left(1 \leq i \leq 4 m_{1}-2\right)$ 
$f_{1}^{\prime}$ in siflrları, $\alpha_{i 2} \neq 0\left(1 \leq i \leq 4 m_{2}-2\right) f_{2}$ 'in siflrları, $\ldots, \alpha_{i n} \neq 0$ $\left(1 \leq i \leq 4 m_{n}-2\right) f_{n}^{\prime}$ in sifirları olsun. O zaman,

$\theta_{5}^{\prime}=\left[\min \left(a_{0}, b_{0}, \sqrt{2} \cdot c_{0}, \sqrt{2} \cdot d_{0}\right)\right]^{2 n} \cdot \frac{\prod_{i=1}^{4 m_{1}-2}\left|\alpha_{i 1}\right| \cdot \prod_{i=1}^{4 m_{2}-2}\left|\alpha_{i 2}\right| \ldots \prod_{i=1}^{4 m_{n}-2}\left|\alpha_{i n}\right|}{d^{4 m_{1}+4 m_{2}+\ldots .+4 m_{n}}} \cdot \frac{1}{2^{4 m_{1}-2}} \cdot \frac{1}{2^{4 m_{2}-2}} \ldots \frac{1}{2^{4 m_{n}-2}}$ olmak üzere

$$
M_{f_{1} \cdot f_{2}} \cdots f_{n} \geq \theta_{5}^{\prime} \cdot M_{f_{1}} \cdot M_{f_{2}} \ldots M_{f_{n}} \operatorname{dir} .
$$

\section{3. $|a|$ 'ya ve Polinomların Sıfırlarına Bağlı Eşitsizlikler}

\section{(Inequalities Depending on $|a|$ and on the zeros of Polynomials)}

Bu alt kesimde $D$ bölgesinde $U T_{2,2}^{4} P(a)$ sınıfı için hem $|a|$ 'ya hem de polinomların köklerine bağlı olarak eşitsizlikler elde edeceğiz.

Teorem 5. $D$ Bölgesinde $4 m$ ve $4 n$ sirasiyla $f, g \in U T_{2,2}^{4} P(a)$ polinom fonksiyonlarının dereceleri ve $f(\mp a)=\mp a^{p}, \quad g(\mp a)=\mp a^{q}$, $\forall \rho_{1} \neq 0 \quad ; \quad \alpha_{i} \neq 0 \quad(1 \leq i \leq 4 m-2) \quad f^{\prime}$ nin siflrları, $\beta_{i} \neq 0 \quad(1 \leq j \leq 4 n-2)$ $g$ 'nin sıfırları olsun. Bu halde,

$$
\theta_{6}=\left[\min \left(a_{0}, b_{0}, \sqrt{2} \cdot c_{0}, \sqrt{2} \cdot d_{0}\right)\right]^{4} \cdot \frac{\max \left(|a|^{p+q-4}\right.}{\left.\prod_{i=1}^{4 m-2}\left|\alpha_{i}\right| \cdot \prod_{j=1}^{4 n-2}\left|\beta_{j}\right|\right)} d^{4 m+4 n} \cdot \frac{1}{2^{4 m-2}} \cdot \frac{1}{2^{4 n-2}} \text { olmak }
$$
üzere,

$$
M_{f . g} \geq \theta_{6} \cdot M_{f} \cdot M_{g} \quad \operatorname{dir}
$$

$$
\begin{aligned}
& \text { Ispat. Teorem } 1^{\prime} \text { in (9) eşitsizliği } \\
& M_{f . g}=\max _{z \in \partial D}|f(z) \cdot g(z)| \geq\left(\min \left(a_{0}, b_{0}, \sqrt{2} \cdot c_{0}, \sqrt{2} \cdot d_{0}\right)\right)^{4} \cdot|a|^{p-2} \cdot|a|^{q-2} \text { ve Teorem } 3^{\prime} \text { in }
\end{aligned}
$$

(15) eşitsizliği

$$
M_{f \cdot g}=\max _{z \in \partial D}|f(z) \cdot g(z)| \geq\left[\min \left(a_{0}, b_{0}, \sqrt{2} \cdot c_{0}, \sqrt{2} \cdot d_{0}\right)\right]^{4} \cdot \prod_{i=1}^{4 m-2}\left|\alpha_{i}\right| \cdot \prod_{j=1}^{4 n-2}\left|\beta_{j}\right|
$$

biçimindedir. O zaman,

$$
M_{f . g}=\max _{z \in \partial D}|f(z) \cdot g(z)| \geq\left[\min \left(a_{0}, b_{0}, \sqrt{2} \cdot c_{0}, \sqrt{2} \cdot d_{0}\right)\right]^{4} \cdot \max \left(|a|^{p+q-4}, \prod_{i=1}^{4 m-2}\left|\alpha_{i}\right| \cdot \prod_{j=1}^{4 n-2}\left|\beta_{j}\right|\right)
$$

olur. Bundan sonra (8) (ya da (16)) göz önüne alınırsa istediğimizi elde ederiz.

Teorem 6. (Genelleştirilmiş Teorem 5) $n \geq 2$ olsun. $D$ Bölgesinde $4 m_{1}, 4 m_{2}, \ldots, 4 m_{n}$ sirasıyla $f_{1}, f_{2}, \ldots, f_{n} \in U T_{2,2}^{4} P(a)$ polinom fonksiyonlarının dereceleri ve $f_{i}(\mp a)=\mp a^{q_{i}}, \forall \rho_{1} \neq 0(i=1,2, . ., n) ; \quad \alpha_{i 1} \neq 0\left(1 \leq i \leq 4 m_{1}-2\right)$ $f_{1}^{\prime}$ in siflrları, $\alpha_{i 2} \neq 0\left(1 \leq i \leq 4 m_{2}-2\right) \quad f_{2}^{\prime}$ in siflrları, $\ldots, \alpha_{i n} \neq 0$ , $\left.1 \leq i \leq 4 m_{n}-2\right)$ f $f_{n}^{\prime}$ in sıfırları olsun. O zaman, 


$$
\begin{gathered}
\theta_{7}=\left[\min \left(a_{0}, b_{0}, \sqrt{2} \cdot c_{0}, \sqrt{2} \cdot d_{0}\right)\right]^{2 n} \cdot \frac{\max \left(|a|^{q_{1}+q_{2}+\ldots+q_{n}-2 n}, \prod_{i=1}^{4 m_{1}-2}\left|\alpha_{i 1}\right| \cdot \prod_{i=1}^{4 m_{2}-2}\left|\alpha_{i 2}\right| \ldots \prod_{i=1}^{4 m_{n}-2}\left|\alpha_{i n}\right|\right.}{d^{4 m_{1}+4 m_{2}+\ldots .+4 m_{n}}} \\
\cdot \frac{1}{2^{4 m_{1}-2}} \cdot \frac{1}{2^{4 m_{2}-2}} \ldots \frac{1}{2^{4 m_{n}-2}}
\end{gathered}
$$

olmak üzere

$$
M_{f_{1} \cdot f_{2}} \cdots f_{n} \geq \theta_{7} \cdot M_{f_{1}} \cdot M_{f_{2}} \ldots M_{f_{n}} \operatorname{dir} .
$$

\section{4. Özel Durum: Bir Birim Ful Hiperbolik Bölge Durumu (Special Case: Case A Unit Full Hyprbolical Region)}

$\mathrm{Bu}$ kesimde bir birim ful hiperbolik bölgede $U T_{2,2}^{4} P(a)$ sınıfı için maksimun modül eşitsizlikleri vereceğiz.

$D$ Bölgesinde $a_{0}=b_{0}=c_{0}=d_{0}=1$ koymakla elde ettiğimiz ful hiperbolik bölge Tanım 2 gereğince bir birim ful hiperbolik bölgedir. Bu bölgeyi $D(1)$ ile gösterelim. $D(1)$ bölgesi için $\min \left(a_{0}, b_{0}, \sqrt{2} \cdot c_{0}, \sqrt{2} \cdot d_{0}\right)=1$, ve $2 d=2 \sqrt{3}$ ya da $d=\sqrt{3}$ dir. $a \in \bar{D}$ noktası $D(1)$ bölgesinde birim çember üzerinde olsun. O zaman, sırasıyla

- $\min (p, q) \geq 3$ ise, (5) Formülünden

$$
M_{f . g} \geq\left(\frac{1}{\sqrt{3}}\right)^{4 m+4 n} \cdot \frac{1}{2^{4 m-2}} \cdot \frac{1}{2^{4 n-2}} \cdot M_{f} \cdot M_{g}
$$

Formülü,

$$
\text { - } p=q=1 \quad(0,-a, a \text { noktaları sabit noktalar }) \text { ya da } \min (p, q)=1
$$
ise, (6) ya da (7) Formülünden

$$
M_{f . g} \geq\left(\frac{1}{\sqrt{3}}\right)^{4 m+4 n} \cdot \frac{1}{2^{4 m-1}} \cdot \frac{1}{2^{4 n-1}} \cdot M_{f} \cdot M_{g}
$$

Formülü,

$$
\begin{gathered}
\cdot \min \left(q_{1}, q_{2}, \ldots, q_{n}\right) \geq 3 \text { ise, (10) Formülünden } \\
M_{f_{1} \cdot f_{2} \cdots f_{n}} \geq\left(\frac{1}{\sqrt{3}}\right)^{4 m_{1}+4 m_{2}+\ldots+4 m_{n}} \cdot \frac{1}{2^{4 m_{1}-2}} \cdot \frac{1}{2^{4 m_{m}-2}} \ldots \frac{1}{2^{4 m_{n}-2}} \cdot M_{f_{1}} \cdot M_{f_{2}} \ldots M_{f_{n}}
\end{gathered}
$$

Formülü,

$$
\text { - } q_{1}=q_{2}=\ldots=q_{n}=1 \quad(0,-a, a \text { noktaları sabit noktalar }) \text { ya da }
$$
$\min \left(q_{1}, q_{2}, \ldots, q_{n}\right)=1$ ise, (11) ya da (12) Formüllerinden

$$
M_{f_{1} \cdot f_{2}} \cdots f_{n} \geq\left(\frac{1}{\sqrt{3}}\right)^{4 m_{1}+4 m_{2}+\ldots+4 m_{n}} \cdot \frac{1}{2^{4 m_{1}-1}} \cdot \frac{1}{2^{4 m_{m}-1}} \ldots \frac{1}{2^{4 m_{n}-1}} \cdot M_{f_{1}} \cdot M_{f_{2}} \ldots M_{f_{n}}
$$

Formülü,

Eğer $j \leq n$ olmak üzere $f_{1}, f_{2}, \ldots, f_{j}$ polinom fonksiyonları için $\min \left(q_{1}, q_{2}, \ldots, q_{j}\right) \geq 3$, geriye kalan $n-j$ tane $f_{j+1}, f_{j+2}, \ldots, f_{n}$ polinom fonksiyonu için $\min \left(q_{j+1}, q_{j+2}, \ldots, q_{n}\right)=1$ ise, (13) Formülünden aşă̆ıda (22) ile numaralayaçağımız 
$M_{f_{1} \cdot f_{2}} \cdots f_{n} \geq\left(\frac{1}{\sqrt{3}}\right)^{4 m_{1}+4 m_{2}+\ldots+4 m_{n}} \cdot \frac{1}{2^{4 m_{1}-2}} \cdot \frac{1}{2^{4 m_{m}-2}} \ldots \frac{1}{2^{4 m_{j}-2}} \cdot \frac{1}{2^{4 m_{j+1}-1}} \cdots \frac{1}{2^{4 m_{n}-1}} \cdot M_{f_{1}} \cdot M_{f_{2}} \ldots M_{f_{n}}$ Formülü, elde edilir.

\section{SONUÇ VE ÖNERILER (RESULTS AND RECOMMENDATIONS )}

$U T_{2,2}^{4} P(a)$ sınıfinın a) $\rho_{1}=0$, b) $\rho_{1}=\rho_{2}=0, \ldots$ c) $\rho_{1}=\rho_{2}=\ldots=\rho_{l}=0$ ( $l \leq m$ ) olan elemanları için de $B_{2}$ ve $D$ hiperbolik bölgelerinde (5)--(22) Formüllerine benzer formüller elde edilebilir.

$U T P(a)$ içinde $U T_{3,1}^{4} P(a), U T_{1,3}^{4} P(a), U T_{4,0}^{4} P(a)$ ile tanımlı sınıflar için de $B_{2}$ ve $D$ hiperbolik bölgelerinde (5)---(22) Formüllerine benzer formüller elde edilebilir.

$$
\begin{array}{r}
z=0 \text { noktasını kök kabul etmeyen } f_{1}(z)=\prod_{i=1}^{m_{1}}\left(z-\alpha_{i_{1}}\right)\left(\alpha_{i 1} \neq 0,1 \leq i \leq m_{1}\right), \\
f_{2}(z)=\prod_{i=1}^{m_{2}}\left(z-\alpha_{i_{2}}\right) \quad\left(\alpha_{i 2} \neq 0,1 \leq i \leq m_{2}\right), \ldots, f_{n}(z)=\prod_{i=1}^{m_{n}}\left(z-\alpha_{i_{n}}\right) \quad\left(\alpha_{i_{n}} \neq 0,1 \leq i \leq m_{n}\right)
\end{array}
$$

formunda $U T_{2,2}^{4} P(a)$ sınıfının elemanı olmayan $n \geq 2$ polinom fonksiyonu verilsin. O zaman, çalışmada yaptığımız ispat yolu izlenerek,

$$
\delta=\frac{\prod_{i=1}^{m_{1}}\left|\alpha_{i 1}\right| \cdot \prod_{i=1}^{m_{2}}\left|\alpha_{i 2}\right| \ldots \prod_{i=1}^{m_{n}}\left|\alpha_{i n}\right|}{d^{m_{1}+m_{2}+\ldots+m_{n}}} \cdot \frac{1}{2^{m_{1}}} \cdot \frac{1}{2^{m_{2}}} \ldots \frac{1}{2^{m_{n}}} \text { olmak üzere }
$$

$M_{f_{1} \cdot f_{2}} \cdots f_{n} \geq \delta \cdot M_{f_{1}} \cdot M_{f_{2}} \ldots M_{f_{n}}$ formülü bulunabilir.

$z=0$ noktasını sırasıyla $k_{1}, k_{2}, \ldots, k_{n}$ katlı kök kabul eden

$$
f_{1}(z)=z^{k_{1}} \cdot \prod_{i=1}^{m_{1}-k_{1}}\left(z-\alpha_{i 1}\right) \quad\left(\alpha_{i 1} \neq 0,1 \leq k_{1} \leq m_{1}\right), \quad f_{2}(z)=z^{k_{2}} \cdot \prod_{i=1}^{m_{2}-k_{2}}\left(z-\alpha_{i_{2}}\right)
$$

$\left(\alpha_{i 2} \neq 0,1 \leq k_{2} \leq m_{2}\right), \ldots, f_{n}(z)=z^{k_{n}} \cdot \prod_{i=1}^{m_{n}-k_{n}}\left(z-\alpha_{i_{n}}\right) \quad\left(\alpha_{i n} \neq 0,1 \leq k_{n} \leq m_{n}\right) \quad$ formunda $U T_{2,2}^{4} P(a)$ sınıfının elemanı olmayan $n \geq 2$ polinom fonksiyonu verilsin. Bu zaman,

$\gamma=\left[\min \left(a_{0}, b_{0}, \sqrt{2} \cdot c_{0}, \sqrt{2} \cdot d_{0}\right)\right]^{k_{1}+k_{2}+\ldots+k_{n}} \cdot \frac{\prod_{i=1}^{m_{1}-k_{1}}\left|\alpha_{i 1}\right| \cdot \prod_{i=1}^{m-k_{2}}\left|\alpha_{i 2}\right| \cdot \prod_{i=1}^{m_{n}-k_{n}}\left|\alpha_{i n}\right|}{d^{m_{1}+m_{2}+\ldots+m_{n}}} \cdot \frac{1}{2^{m_{1}-k_{1}}} \cdot \frac{1}{2^{m_{2}-k_{2}}} \ldots \frac{1}{2^{m_{n}-k_{n}}}$ olmak üzere

$$
\begin{aligned}
& M_{f_{1} \cdot f_{2} \ldots f_{n}} \geq \gamma \cdot M_{f_{1}} \cdot M_{f_{2}} \ldots M_{f_{n}} \text { formülü bulunabilir. } \\
& M(f, R)=\max _{|z|=R}|f(z)| \text { ve } M(f, 1)=\max _{|z|=1}|f(z)| \text { olarak tanımlansın. }
\end{aligned}
$$

$M(f, R)$ ve $M(f, 1)$ arasında var olan eşitsizlik formülleri için [1, 9, 10, 11, 12, 13 ve 17] önerilir. Bir ful hiperbolik bölge ve bu bölgeden 
oluşturulan birim ful hiperbolik bölge için polinomların maksimum modülleri arasında $[1,9,10,11,12,13$ ve 17]'dekilere benzer eşitsizlikler oluşturulabilir.

\section{TARTIŞMALAR (DISCUSSIONS)}

(1), (2), (3) ve(4) formüllerindeki katsayılar polinomların köklerinin mutlak değerli olarak en küçük köküne bağlıdırlar. Polinomların köklerini bulmak sorun değilse, bu formülleri uygulamak oldukca kolaylık sağlar. Elbette $U T_{2,2}^{4} P(a)$ sınıfının elemanları için de (1), (2), (3) ve (4) Formüllerini yazabiliriz. $D$ ful hiperbolik bölgesi $B_{2}$ hiperbolik bölgesinden daha genel bir bölgedir. $D$ bölgesinde $U T_{2,2}^{4} P(a)$ sınıfının elemanları için bulduğumuz (5), (6), (7), (10), (11), (12) ve (13) Formüllerindeki katsayılar polinomların köklerinden bağımsızdır. İlgili katsayılar $|a|$ 'ya (ya da kuvvetine) bağlıdır ve sınıf verildiğinde $|a|$ bellidir. O zaman elde ettiğimiz (5), (6), (7), (10), (11), (12) ve (13) Formülleri oldukça kullanışlıdır.

Bir birim ful hiperbolik bölge daima birim diski içerir. $U T_{2,2}^{4} P(a)$ sınıfının elemanları için, birim diskte [17], [15], [14], [7] ve [3]'de verilen eşitsizliklerdeki katsayılar geçerli iken, aynı polinom fonksiyonlar için $D(1)$ birim ful hiperbolik bölgesinde(18), (19), (20), (21) ve (22) Formüllerindeki katsayılar geçerlidir. Tersine tartışmalar da yapılabilir. Örneğin, $D(1)$ birim ful hiperbolik bölgesi $d=R=\sqrt{3}$ yarıçaplı daire tarafından kapsanır.

\section{KAYNAKLAR (REFERENCES)}

1. Ankeny, N.C. and Rivlin, T.J., (1955). On a theorem of S. Brenstein, Pasific J. Math., ss:849-852.

2. Aziz, A., (1987). Growty of polynomials whose zeros are within or outside a circle, Bull. Austral.Math. Soc. Vol.35, ss:247-256.

3. Çelik, A., (2013). Inequalities for polynomial functions, NWSAPhysical Sciences, Volume: 8, Number: 2, ss:32-47. DOI URL http://dx.doi.org/10.12739/NWSA.2013.8.4.3A0064

4. Çelik, A., (2012). New inequalities for Maximum Modulus Values of polynomial functions, Hacettepe Journal of Mathematics and Statistics, Volume: 41 (2), ss:255-263.

5. Çelik, A., (2009). On the ünivalent functions with three preasigned values and automorphism of an open disk, NWSAPhysical Sciences, Volume: 4, Number: 1, ss:36-41,2009.

6. Çelik, A., (2004). Maximum module values of polynomials on $|z|=R$ $(R>1)$, Üniv. Beograd, publ. Elektrotehn. Fak., ser.Mat.15, ss:1-6

7. Çelik, A., (1997). A note on Mohr's paper, Üniv.Beograd, publ. Elektrotehn. Fak., ser. Mat.8, ss: 51-54

8. Deshpande, J.V., (1986). Complex Analysis (Tata MCGraw-Hill Publising Company, New Delhi).

9. Dewan, K.K. and Ahuja, A., (2011). Growty of polynomials havings zeros inside a circle, Int. Journal of Math. Analysis, Volume:5, no.11, ss:499-505.

10. Gardner, R.B., (2004). Some results conserning rate of Growty of polynomials, East Journal on approximations, Volume: 10, Number: 3, ss:301-312. 
11. Gardner, R.B., Govil, N.K., and Musukula, S.R., (2005). Rate of Growty of polynomials not vanishings inside a circle, Journal of Inequalities in Pure and Applied Mathematics, Volume: 6, Issue: 2, Articale: 53, ss:1-21.

12. Milonovic' G.V., Mitrinovic' D.S., and Rassias, M.TH., (1994). Extremal Problems, Inequalities Zeros (ord Scientific Publ. Co., Singapore, New Jersey, London.

13. Mir, A., Devan, K.K., and Sing, N., (2009). Some inequalities concerning the rate of growty of polinomials, Turk. J. Math., 33, ss:239-247.

14. Mohr, E., (1992). Bemerkung Zu der arbeit Van A.M. Ostrowski Notiz Uber Maximalwerte von polynomen auf dem einheitskreis Üniv. Beograd, publ. Elektrotehn. Fak, ser. Mat.3, ss: 3-4.

15. Ostrowski, A.M., (1979). Notiz uber Maximalwerte von polynomen auf dem einheitskreis, Üniv. Beograd, publ.Elektrotehn. Fak., ser. Mat. Fiz., No 34-637, ss: 55-56.

16. Quazi, M.A., (1992). On the maximum module values of polynomials, Proceding of the American Mathematicle Soc., Volume: 115, Number: 2, ss:337-343.

17. Rassias, M.T.H., (1986). A new inequality for complex-valued polynomial functions, Proc. Amer. Math. Soc. 9, ss: 296-298. 